\title{
L'enseignement de l'anatomie à Lyon: un exemple d'évaluation de programme
}

\author{
Éric J. VOIGLIO ${ }^{1,2,3,}$ Benoît RATTINI I,2, François MITHIEUX'2, Gualter VAZ ${ }^{2,}$ Jean-Christophe LIFANTE,
} Frédéric RONG RAS $^{2,}$ Jean-Pierre H. NEDHARDT ${ }^{1,2}$ André MORIN²

\begin{abstract}
RésuméContexte: En raison dela réforme de la maitrise de sciences biologiques et médicales (M SBM ), un certificat optionnel d'anatomie appliquée a éée créè à la Faculté de M édecine Lyon-N ord pour l'année 1997-98. L'année suivante (1998-99), en raison de son succès, ce certificat optionnel a évoluévers un certificat deD i plômePréparatoireà la Recherche Biomédicale(D PRBm). But: Afin d'identifier de manière objective les défauts, deles corriger et d'améliorer ains la qualitéde l'enseignement, uneévaluation de programme a éé effectuée sur les deux années universitaires M éthode Un questionnaire anonyme a été remis aux éudiants. Ces derniers devai ent noter de 0 à 10 cinq critères (conditions matérielles assiduité des étudiants, assiduité des enseignants, intérêt perçu, qualité perçue) pour chacun des six types d'enseignement (cours magistraux, enseignements dirigés, dissections, enseignements dirigés d'informatique, préparation des exposés au tableau et tutorat pour la préparation des mémoi res). Lesquestionnaires ont été récupérés avant la prodamati on des résultats des épreuves Résultats La première année, 22 étudiants sur 30 (73\%) ont rendu le questionnaire. La deuxième année, 31 étudiantssur 31 (100\%) ont rendu le questionnaire Cette étude a permis notamment d'identifier uneinsuffisance dans les conditions matérielles $(5,35)$ et l'encadrement des dissections $(3,43)$ qui a pu être corrigée l'année suivante $(6,25$ et 7,50$)$. Les deux meilleures notes pour l'intérêt perçu ont été obtenues par les di ssections $(8,0$ et 8,61$)$ et la préparation aux exposés au tableau $(7,45$ et 8,8$)$. Les meilleures notes pour la qualitéperçueont ééattribuées aux enseignements dirigés d'informatique $(8,09$ et 8,42). Conclusion: L'anatomie est une di scipline très appréciée des étudiants Uneévaluation de programmea permis d'identifier les pointsfaibles et de les corriger ou de solliciter desmoyensà cettefin.
\end{abstract}

Mots ClésAnatomie humaine; enseignement médical; évaluation de programme.

Summary context: Because of the reform of the master's in biologic and medical sciences (M BM S), an optional applied anatomy certificate was created at the Faculty of M edicine Lyon-N ord for the academic year 1997-98. Thefollowing year (1998-99) in light of its success this certificate evolved as a Preparatory Diploma in Biomedical Research (PD BR). Goal: To identify objectively and to correct theweaknesses in order to improve the quality of teaching, a program evaluation on both academic years was performed. M ethod:An anonymous questionnaire was distributed to the students They had to evaluate five criteria on a 10-point scale (0-10) (material conditions, assiduity of students, assiduity of teachers, perceived interest and perceived quality) for each of sx teaching methods (lectures, group teaching, di ssections, group teaching in informatics, preparation of blackboard talks and tutorialsfor dissertation). Q uestionnaireswere collected beforepublication of examination results Results:T he $1^{ \pm}$year, 22 students out of 30 (73\% ) sent back the questionnai re), the $2^{\text {nd }}$ year, 31 sudents out of 31 (100\%) sent back the questionnaire This study permitted mainly the identification of insufficiency of material conditions (5.35) and supervision of dissections (3.43) which have been corrected the following year (6.25 and 7.50). The best two marks concerning perceived interest were given to dissections ( 8.0 and 8.61) and to preparing of blackboard talks (7.45 and 8.8). The bet marks for perceived quality were given to group teaching in informatics (8.09 and 8.42). Condusion:Anatomy is a disciplinehighly appreciated by medical students. A program evaluation allowed to identify weak points and to correct them or to have an input on means to reach this purpose

KeywordSH uman anatomy; medical teaching; program evaluation.

Pédagogie M édicale $2002 ; 3: 27-32$

1. Service de Chirurgie d'Urgence - Centre Hospitalier Lyon-Sud. 2. Laboratoire d'Anatomie Médico-Chirurgicale - Facultés de Médecine Lyon-Nord et Grange Blanche - Université Claude Bernard-Lyon I. 3. UMRETIE - Unité Mixte de Recherche Éoidémiologique Transport Travail Environnement.

Correspondance : Docteur Eric J. Voiglio - Laboratoire d'Anatomie Médico-Chirurgicale - Université Claude Bernard-Lyon I Faculté de Médecine Lyon-Nord - Domaine Rockefeller - F69373 LYON Cedex 08 - France - Tél. +33 (0)478 861412 mailto: voiglio@rockefeller.univ-lyon1.fr 


\section{Concepts et Innovations}

\section{Introduction}

En France, depuis 1987 les étudiants en médecine avaient la possibilité de suivre parallèlement à leurs études médicales un cursus de sciences biologiques et médicales dont la validation aboutissait à l'obtention d'une maîtrise de sciences biologiques et médicales $(\mathrm{M} \mathrm{SBM})^{1}$. L'obtention de la maîtrise était subordonnée à la validation de trois certificats, la réussite à l'internat de spécialité comptant pour un certificat. $D$ ans toutes les facultés de médecine de France, les laboratoires d'anatomie s'étaient investis dans cet enseignement, et délivraient le certificat d'anatomie et organogen èse et des certificats d'an atomie spécialisée (biomécanique, neuro-anatomie, angio-anatomie, anatomie chirurgicale, radio-anatomie, etc.).

En 1992, une réforme des études médical es ${ }^{2}$ faisait obligation aux étudiants inscrits en deuxième et troisième années de médecine de suivre un enseignement complémentaire de 80 à 100 heures. Les certificats de M SBM faisaient partie des enseignements complémentaires offerts aux étudiants. Ces certificats, toutes disciplines confondues, voyaient alors leur effectif augmenter considérablement, transformant ainsi un enseignement destiné initialement à un petit nombre en enseignement de masse.

Afin de pallier cet inconvénient, en 1997 une réforme de la $\mathrm{MSBM}^{3}$ entraînait une limitation notable des capacités d'accueil des étudiants. En raison du faible nombre des certificats optionnels existant à la rentrée 1997, un certificat optionnel d'anatomie appliquée ( $C O A A)$ a été créé à la Faculté de $M$ édecine LyonN ord pour I'année 1997-1998. C et enseignement comportait 20 heures de cours magistraux, 20 heures d'enseignement dirigé, 20 heures de dissections et 40 heures pour la rédaction d'un mémoire (tableau 1 ). L'examen final comportait une épreuve de Q CM (coefficient 1), un exposé oral de 10 minutes au tableau sur un sujet anatomique choisi par l'étudiant (coefficient 1), une dissection tirée au sort de 3 heures commentée en 5 minutes (coefficient 1 ) et une soutenance de mémoire (coefficient 2 ).

L'année suivante (1998-1999), en raison de son succès, ce certificat optionnel a évolué vers un certificat de Diplôme Préparatoire à la Recherche Biomédicale (D PRBm) ${ }^{3}$, en complétant le programme par huit cours magistraux (tableau 2). Les étudiants étai ent

\section{Tableau 1 : Programme du Certificat O ptionnel d'Anatomie Appliquée (COAA).}

Cours magistraux ( 2 heures):

Prévention du risque infectieux au cours des dissections chirurgicales

Biomécanique du thorax

Internet: mode d'emploi

$M$ éthodes en histologie et en embryologie

Laparotomies, éventrations, hernies

Recherche chirurgicale: comment réaliser un projet

Anatomie appliquée aux interventions de chirurgie digestive courantes

Traumatismes de la face

Fractures du rachis: mécanismes,

biomécanique, traitement

Biomécanique des fixateurs externes, bases anatomiques de la pose

Enseignements dirigés ( 2 heures) :

Sutures des plaies

Radiologie et tomodensitométrie du thorax

Exposé oral (1)

Exposé oral (2)

Internet

Tomodensitométrie abdomino-pelvienne

Logiciels courants: Power point ${ }^{\circledR}$ et Word ${ }^{\circledR}$

Interprétation des artériographies

Examen neurologique dans les fractures

de la colonne vertébrale

Traumatismes des membres

$D$ issections (2 heures):

Techniques de dissection

Région supracardiaque

Région inguinale

Pédicule hépatique

Rein

Creux axillaire

Pli du coude, canal carpien

Trigone fémoral

Région carotidienne

Dissection libre 


\section{Tableau 2: Cours spécifiques du DPR Bm (en complément aux cours du COAA).}

Cours magistraux (2 heures)

Introduction à l'étude des monstruosités

et malformations

Evolution, fermeture et anomalies du tube neural

Les cél osomies

Adaptation du squelette à la bipédie (I)

Adaptation du squel ette à la bipédie (II)

Les monstres doubles

O rganogenèse et malformations des voies biliaires extra-hépatiques

O rganogenèse et anomalies du système porte hépatique.

soumis aux mêmes modalités d'examen que l'année précédente.

Tyler en 1949 a introduit les procédures d'évaluation comme principe indispensable dans toute démarche pédagogique $e^{4}$. M adaus en 1983 a défini les quatre points d'une évaluation: contexte, intrants, processus et produit ${ }^{5}$. Le contexte était imposé (comme souvent en France) par une nécessité administrative, et les intrants avaient déjà été évalués par les commissions de pédagogie et conseils des facultés concernées.
L'évaluation du produit relevait de l'examen final composé de quatre épreuves destinées à évaluer le savoir (QCM), le savoir faire (dissection, mémoire, exposéau tableau) et le savoir être (mémoire, exposéau tableau) des étudiants. Restait donc à évaluer le processus afin d'identifier de manière objective les défauts, de les corriger et d'améliorer ainsi la qualité de l'enségnement. Une évaluation de programme a ainsi été effectuée à la fin des deux années universitaires.

\section{Matériel et méthodes}

A la fin de l'année universitaire, un questionnaire anonyme a été remis aux étudiants. Ces derniers devaient noter de 0 à 10 cinq critères (conditions matérielles, assiduité des étudiants, assiduité des enseignants, intérêt perçu, qualité perçue) pour chacun des six types d'enseignement (cours magistraux, enseignements dirigés, dissections, enseignement dirigés d'informatique, préparation des exposés au tableau noir et tutorat pour la préparation des mémoires) (figure 1). Les questionnaires ont été récupérés avant la proclamation des résul tats des épreuves. Les moyennes et les écarts types par critère ont été calculés pour chaque type d'enseignement. Le test $t$ de Student-Fisher a été utilisé pour comparer les moyennes.

\section{Figure 1: G rille d'évaluation}

M erci de noter de 0 à 10 chaque critère d'évaluation :

\begin{tabular}{l|l|l|l|l|l}
\hline $\begin{array}{l}\text { Cours } \\
\text { magistraux }\end{array}$ & $\begin{array}{c}\text { Conditions } \\
\text { matérielles }\end{array}$ & $\begin{array}{c}\text { Votre } \\
\text { assiduité }\end{array}$ & $\begin{array}{c}\text { Assiduité des } \\
\text { enseignants }\end{array}$ & $\begin{array}{c}\text { Intérêt } \\
\text { perçu }\end{array}$ & $\begin{array}{c}\text { Qualité } \\
\text { perçue }\end{array}$ \\
\hline $\begin{array}{l}\text { Enseignements } \\
\text { dirigés d'anatomie }\end{array}$ & & & & \\
\hline $\begin{array}{l}\text { Dissections } \\
\text { anatomiques }\end{array}$ & & & & & \\
\hline $\begin{array}{l}\text { Enseignements } \\
\text { dirigés à la } \\
\text { médiathèque }\end{array}$ & & & & & \\
$\begin{array}{l}\text { Préparation aux exposés } \\
\text { aux tableaux } \\
\begin{array}{l}\text { Tutorat pour la } \\
\text { préparation } \\
\text { des mémoires }\end{array}\end{array}$ & & & & & \\
\hline
\end{tabular}




\section{Concepts et Innovations}

\section{Résultats}

La première année, 22 étudiants sur 30 (73\%) ont rendu le questionnaire. La deuxième année, 31 étudiants sur 31 $(100 \%)$ ont rendu le questionnaire. Les résultats sont représentés sous forme d'histogrammes (moyennes \pm écarts types) dans la figure 2.

En ce qui concerne les conditions matérielles, la note moyenne la plus basse a été obtenue pour les dissections, avec toutefois une amélioration l'année suivante (5,35 et $6,26)$. La meilleure note concernant les conditions maté rielles a été obtenue les deux années pour les enseignements dirigés d'informatique à la médiathèque. L'assiduité des étudiants a toujours été bonne, et la note la plus basse $(7,37)$ a été obtenue la deuxième année, pour la préparation aux exposés oraux.

En ce qui concerne l'assiduité des enseignants, une (très) mauvaise note a été obtenue la première année pour l'encadrement des dissections $(3,43)$; ce point a été corrigé efficacement l'année suivante (note de 7,50 attribuée par les étudiants, $p<0,001)$.

Pour l'intérêt perçu par les étudiants, les deux meilleures notes ont été obtenues la première annéepar les dissections $(8,0)$ et la préparation aux exposés au tableau noir $(7,45)$. L'année suivante, les deux premières notes ont été attribuées à la préparation aux exposés au tableau $(8,8)$ et aux dissections (8,61). Q uant à la qualité perçue, les meilleurs notes ont été attribuées les deux années aux enseignements dirigés d'informatique $(8,09$ \& 8,42$)$.

\section{Discussion}

\section{Critique de la méthodologie}

La confidential ité des données était assurée par un questionnaire anonyme placé dans une enveloppe. Une telle confidentialité est évidemment indispensable afin d'améliorer l'objectivité. La première année, il avait été demandé aux étudiants de ramener le questionnaire distribué lors de l'épreuve écrite au secrétariat; seulement 23 étudiants sur 30 ont rendu le questionnaire. L'année suivante, les étudiants devai ent rendre le questionnaire au moment de l'épreuve orale et une exhaustivité de $100 \%$ a pu être obtenue.

L'échelle numérique utilisée comportait 10 points: 0 = inacceptable ; 1 = mauvais; [1 à 3] = médiocre; [3 à 6] = moyen ; [6 à 8] = bon ; 9 = excellent; 10 =exceptionnel. Cetype d'échelle a déjà été utilisé dans notre faculté pour des évaluations de l'enseignement ${ }^{6}$.

\section{Identification et correction des dysfonctionnements}

Une telle évaluation de l'enseignement permet d'identifier les dysfonctionnements et de les corriger.

Le poste qui retenait immédiatement I'attention était celui des dissections, tant par les conditions matérielles (note la plus basse tous types d'enseignements confondus) que par l'assiduité des enseignants (note de 3,43 ). Les conditions matérielles sont, à Lyon, celles d'un laboratoire du début du $x x^{e}$ siècle, et il est difficile de les améliorer de façon spectaculaire. En revanche, il a été possible d'améliorer de façon très significative l'encadrement, notamment en recrutant des moniteurs parmi les étudiants qui avaient suivi l'enseignement et qui avai ent été si sévères envers leurs aînés parfois retenus par des obligations hospital ières. D es binômes étudiant-interne ou étudiant-assistant ont parfaitement fonctionné. II est évident que la correction de ce dysfonctionnement est reflétée par une amélioration de la qualité perçue.

Le deuxième poste qui a retenu l'attention était celui du tutorat pour la préparation des mémoires. Un effort d'encadrement, et un renforcement de l'équipement informatique du laboratoire a permis d'améliorer les résultats.

D 'une manière plus générale, les améliorations statistiquement significatives d'une année sur l'autre prouvent que les actions entreprises pour améliorer la qualité ont été fructueuses.

\section{Identification des points forts de l'enseignement}

L'assiduité des étudiants a, tous types d'enseignements confondus, obtenu une note supérieure à 7,3 les deux années. II est à noter qu'aucun contrôle n'était imposé. Ce résultat mérite d'être souligné, à une époque où dans d'autres disciplines on s'interroge sur les astuces à employer pour inciter les étudiants à l'assiduité. 0 n peut donc s'interroger sur ce qui incite un étudiant moyen en médecine, en l'absence de toute contrainte à assister à un enseignement. C'est probablement que les sujets traités I'intéressent, et d'aill eurs les étudiants ont attribué pour l'intérêt perçu une note supérieure à $7 / 10$ à toutes les formes d'enseignement (sauf la première année au tutorat des mémoires). II est toujours possible d'enseigner une anatomie purement descriptive, extrêmement détaillée, et sans aucun rapport avec 
Figure 2 : Moyennes \pm écart type des notes obtenues aux différents items du questionnaire en 1998 (vert) et 1999 (gris).

$$
\star P<0,05 ; \star \star P<0,001
$$
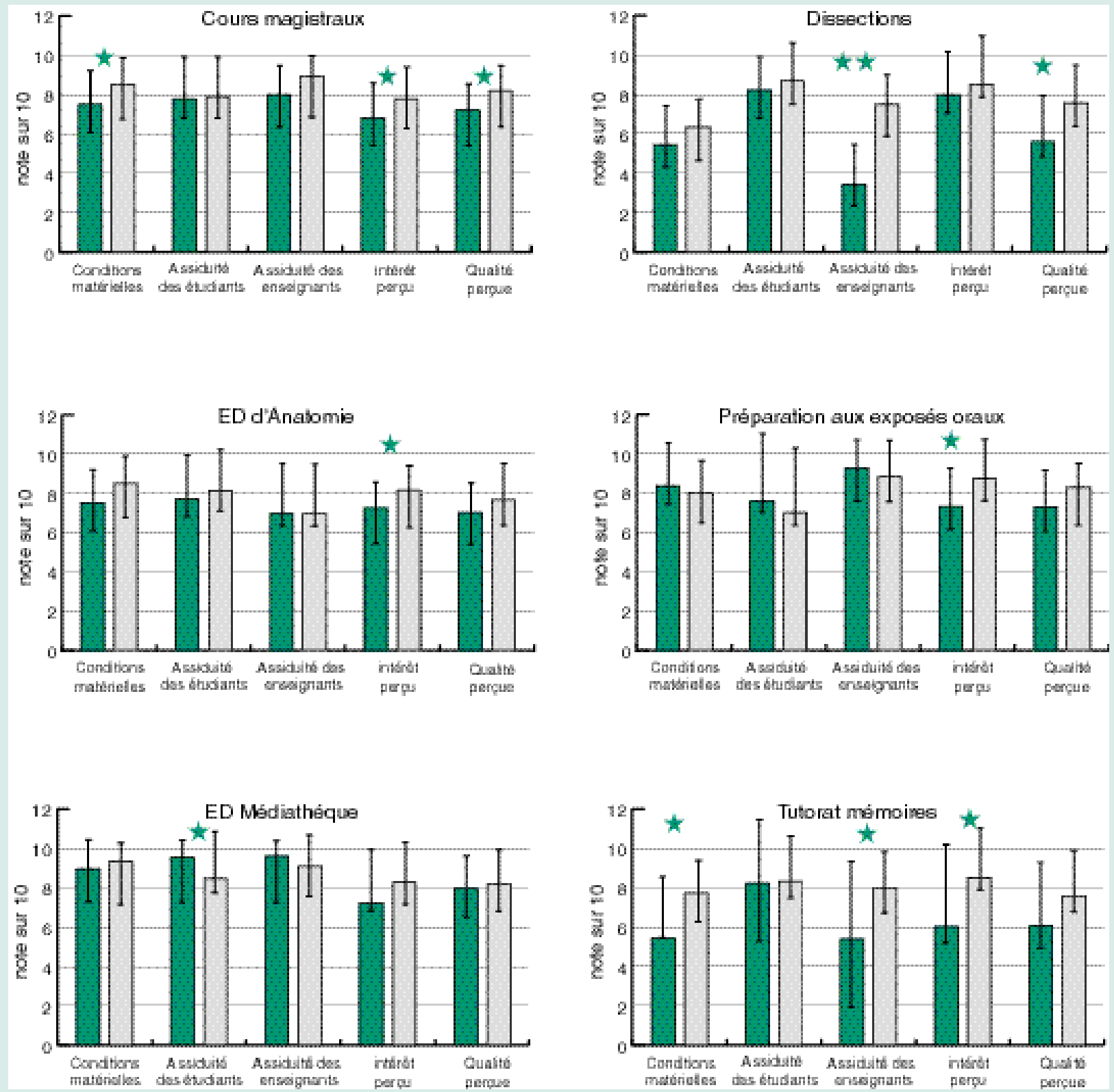


\section{Concepts et Innovations}

I'exercice de la médecine. N ous avons au contrai re pris le parti d'enseigner une anatomie appliquée à la sémiologie, à la pathologie chirurgicale et aux techniques d'imagerie. Ainsi nos étudiants sont conscients qu'ils apprennent leur futur métier de médecin en assistant aux enseignements. N ous sommes même allés plus loin dans ce raisonnement, et nous nous servons de l'anatomie, discipline appréciée des étudiants, pour leur faire acquérir des compétences utiles à leur futur exercice médical, comme l'expression orale en public, la rédaction d'un mémoire, I'utilisation des outils multimédia et de l'Internet, ainsi que l'initiation à la recherche. A I'heure où les dissections anatomiques sont menacées de disparition pour des raisons diverses (manque de corps, réduction des surfaces attribuées à l'anatomie, risque de contagion... ) il est intéressant de noter que les étudiants considèrent leur intérêt fondamental attribuant une note de 8,0 la première année et de 8,6 la deuxième année. Un effort particulier doit donc être fait pour les maintenir dans le cursus des études médicales, et obtenir des crédits pour rénover les laboratoires.

Les étudiants ont autant apprécié la préparation aux exposés au tableau noir qui leur a été donnée que la formation à l'utilisation des outils multimédia. C eci prouve qu'aux yeux des étudiants, les deux méthodes pédagogiques sont complémentaires et non antagonistes. L'introduction des moyens informatiques ${ }^{7,8,9}$ dans I'enseignement de l'anatomie constitue une évolution et non une révolution.

Les étudiants ont pu bénéficier pour les enseignements dirigés d'informatique d'une salle équipée de 12 postes informatiques avec projecteur multimédia et située dans un bâtiment neuf d'agencement très agréable. Les conditions matérielles et la qual ité perçue des enseignements dirigés d'informatique ont obtenu les deux années les meilleures notes. U ne bonne logistique contribue à la qualité de l'enseignement.

\section{Conclusion}

L'anatomie est une discipline très appréciée des étudiants. Son enseignement repose depuis les origines sur des cours magistraux, des dissections, des exposés au tableau noir. Les nouvelles méthodes pédagogiques utilisant l'outil informatique sont aussi appréciées par les étudiants que les autres méthodes pédagogiques. U ne évaluation de programme sur deux ans de ce nouvel enseignement d'anatomie a permis d'identifier les points faibles et de les corriger ou de solliciter des moyens à cette fin.

\section{Références}

1. Arrêté du 24 juin 1987 portant création de la maîtrise de sci ences biologi ques et médicales.

2. Arrêté du 18 mars 1992 relatif à l'organisation du premier cycle et de la première année du deuxième cycle des études médicales, Titre III, Section2, A rt.19.

3. Arrêté du 3 mars 1997 portant création du diplôme préparatoire à la recherche biomédicale.

4. Tyler RW. Basic Principles of Curriculum and Instruction. Chicago: U niversity of Chicago, 1949.

5. M adaus $G$, Scriven $M$, Stufflebeam D. Evaluation models: Viewpoints on educational and human services evaluation. Boston: Kluver-N ijhoff Pub. 1983.

6. Llorca G. Rapport d'évaluation de l'enseignement universitaire à I'U FR L yon-N ord. U niversité Claude Bernard Lyon I, 1997.
7. Voiglio EJ, F rattini $B$, Romeuf $D, M$ orin $A$, $\mathrm{N}$ eidhardt JPH, Laville M. F rench mirror site of the N PAC Visible H uman Viewer: first year evaluation. Surg Radiol Anat 1999; 21(2) :139-41.

8. Frasca $D, M$ alezieux $R, M$ erens $P, N$ eidhardt JPH, Voiglio EJ. Prospecting and evaluation of the anatomy sites on the Internet (update 1999). Surg Radiol Anat 2000; 22: 107-110.

9. Tavares M A, Dinis M achada J, Silva M C. Computer based sessions in radiological anatomy: one year's experience in clinical anatomy. Surg Radiol Anat 2000; 22 : 29-34. 